\title{
Induktive Platinenerwärmung
}

\section{für den Presshärteprozess}

Für die Herstellung von Bauteilen mit konstanten mechanischen Eigenschaften durch das Presshärten wird im Anschluss an das Erwärmen ein homogenes Temperaturprofil in der Platine benötigt. Dieses ist im konventionellen Ofenprozess gegeben, stellt aber bei der induktiven Erwärmung eine besondere Herausforderung dar. Im Laufe eines Projektes ist es Forschern gelungen, eine Methode zur einstufigen induktiven Erwärmung zu generieren, mit der Platinen und somit auch Bauteile mit konstanten mechanischen Eigenschaften hergestellt werden konnten.

Pressgehärtete Bauteile sind in den vergangenen Jahren zunehmend als Strukturelemente im Fahrzeug, beispielsweise bei BSäulen oder auch als Türverstärkungen im Bereich des Seitenaufprallschutzes eingesetzt worden. Dabei findet hauptsächlich Mangan-Bor-Stahl 22MnB5 Anwendung. Das Material soll sich durch hervorragende Festigkeitseigenschaften auszeichnen. Realisiert werden die Festigkeitseigenschaften im Presshärteprozess durch eine Erwärmung auf über $900^{\circ} \mathrm{C}$ mit anschließender Umformung und definierter Abkühlung. Für die Erwärmung werden in der industriellen Praxis hauptsächlich Rollenherdöfen verwendet, deren Substitutionsmöglichkeit durch induktive Erwärmungsanlagen Gegenstand des Forschungsvorhabens war.

Am Lehrstuhl für Leichtbau im Automobil der Universität Paderborn unter der Leitung von Professor Dr. Thomas Tröster wurde dazu das Forschungsvorhaben "Einsatz des induktiven Erwärmens und Ermittlung der entsprechenden Prozessfenster für das Presshärten" mit einer Laufzeit von 30 Monaten durchgeführt und nun erfolgreich abgeschlossen, wie die Fosta - Forschungsvereinigung Stahlanwendung berichtet. Im Laufe des Forschungsprojektes konnten die Wissenschaftler eine Methode zur einstufigen induktiven Erwärmung zu generieren.
Ein wesentlicher Unterschied zwischen der Ofenerwärmung und der induktiven Erwärmung ist die benötigte Aufheizzeit, berichten die Wissenschaftler: Die Verweilzeiten im Strahlungsofen betragen je nach Blechdicke bis zum Erreichen der benötigten Temperatur mehrere Minuten, wohingegen die induktive Erwärmung innerhalb weniger Sekunden erfolgen kann. Zusätzlich zu der Prozessgestaltung der induktiven Erwärmung waren die Untersuchung der mechanischen Eigenschaften induktiv innerhalb weniger Sekunden erwärmter Platinen und die Festlegung von Prozessfenstern Ziele des Projekts.

Zur Prozessfensterbestimmung wurden unterschiedliche Versuchsreihen durchgeführt. Neben der Untersuchung der Auswirkungen unterschiedlicher Erwärmungstemperaturen auf mechanische Eigenschaften wurden die Folgen zusätzlicher Haltezeiten überprüft. Zusammenfassend konnten die Experten feststellen, dass eine Erwärmung des Werkstoffs 22MnB5 auf mindestens $900{ }^{\circ} \mathrm{C}$ innerhalb von 20 s ohne zusätzliche Haltezeit zu mechanischen Eigenschaften führte, die mit der konventionellen Ofenerwärmung vergleichbar waren. Selbst Temperaturerhöhungen auf bis zu $1100{ }^{\circ} \mathrm{C}$ sollen keine wesentlichen Unterschiede zur Folge gehabt haben. Als wesentliche Punkte wurden in diesem Forschungsprojekt eine Methode zur induktiven Erwärmung im Presshärteprozess entwickelt und entsprechende Prozessgrenzen bestimmt. Das IGF-Vorhaben 16319 N (P 805) „Einsatz des induktiven Erwärmens und Ermittlung der entsprechenden Prozessfenster für das Presshärten" der Fosta wurde über die Arbeitsgemeinschaft industrieller Forschungsvereinigungen Otto von Guericke (AiF) im Rahmen des Programms zur Förderung der industriellen Gemeinschaftsforschung und -entwicklung (IGF) vom Bundesministeriums Wirtschaft und Technlogie aufgrund eines Beschlusses des Deutschen Bundestags gefördert.
Massivumformung bedeutet DrNaink

Massivumgeformte Bauteile ermöglichen höchste dynamische Belastungen und reduzieren durch Gewichtsoptimierung Verbrauch und Emission.

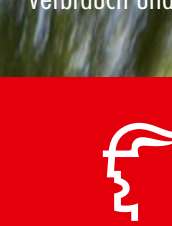

HANNOVER MESSE

8.-I2. APRIL 20I3

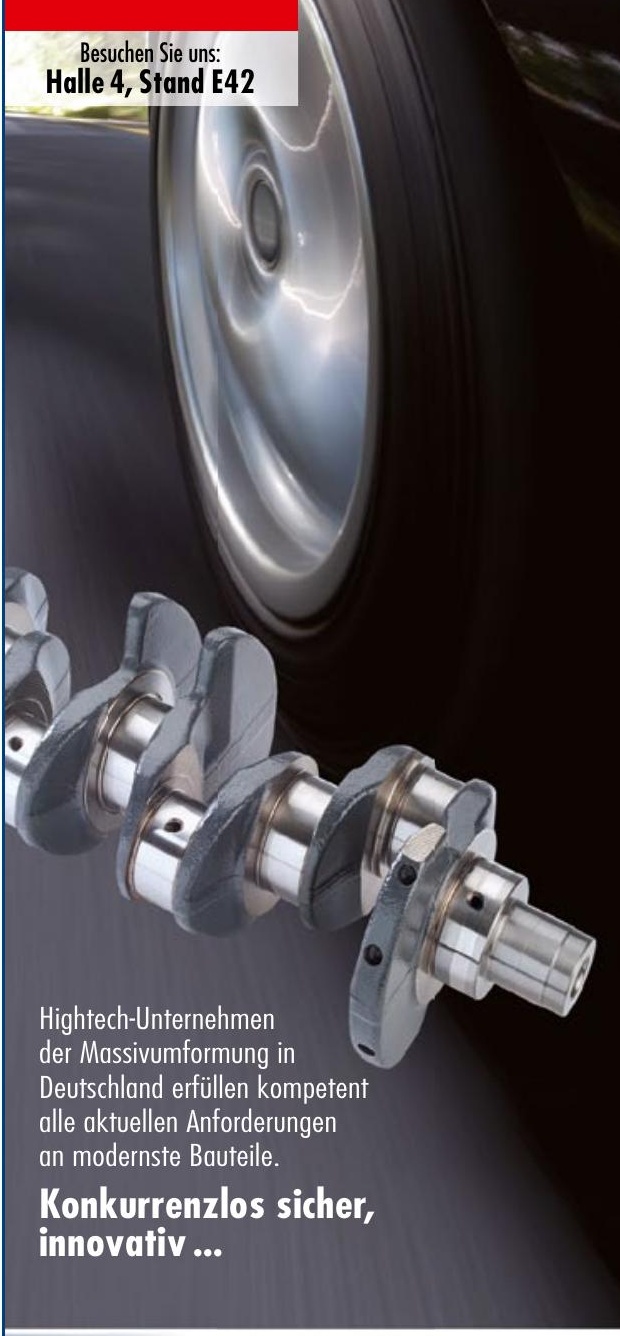

